\title{
PHENIX Experiment Highlights
}

\author{
Alexandre Lebedev for the PHENIX collaboration ${ }^{1, *}$ \\ ${ }^{1}$ Iowa State University
}

\begin{abstract}
Over sixteen yesrs of running the PHENIX experiment at RHIC has collected a wealth of data on proton-proton, proton-nucleus, and nucleusnucleus collisions at various collision energies. In this talk we present the most recent and exciting results from the PHENIX experiment.
\end{abstract}

\section{Introduction}

In 16 years of running, the PHENIX experiment at RHIC has collected an enormous amount of data at 9 different energies for 9 different collision species. Almost two hundred papers were published, 12 of them in the past year (2018).

In this talk we present the most recent and interesting results.

The first section describes the results from the polarized proton collisions, including longitudinal single spin asymmetry of W-bosons, and transverse single spin asymmetry of charged hadrons, $J / \psi$ and $\eta$-mesons.

In the second section we present results from collisions of light nuclei like $\mathrm{p}$ and $\mathrm{d}$ with heavy ions. In particlar, we discuss longitudinal dynamics in small systems, small systems geometry scan, and nuclear modification factors for various particles. At the end of the section we present Drell-Yan and direct thermal photon measurements.

The third section discusses heavy ion results, including suppression of different particle species in heavy ion collisions with an emphasis on strangeness. Charm and bottom flow measurement in $\mathrm{Au}+\mathrm{Au}$ collisions is also described in this section.

\section{Spin physics}

It is well known that proton spin is not a simple sum of valence quark spins. Sea quarks, qluons, and orbital momentum of both quarks and gluons also contribute to it. The PHENIX experiment aims to study both longitudinal spin structure and transverse spin phenomena.

In a recently published paper [1] the PHENIX experiment presented a measurement of longitudinal single spin asymmetry $\left(A_{L}\right)$ for $W$-bosons (see Fig. 1 . The results are in agreement with those from STAR collaboration and global fits.

In conventional pQCD calculations transverse single spin asymmetry $\left(A_{N}\right)$ is expected to be close to zero. Fig. 2 shows transverse single spin asymmetry of charged hadrons produced in $\mathrm{p}+\mathrm{p}$ collisions as a function of $x_{F}=2 p_{T} / \sqrt{s}$ as measured by the PHENIX experiment. While at negative $x_{F} A_{N}$ is consistent with zero, there is a statistically significant deviation at positive $x_{F}$. We observe positive $A_{N}$ for positively charged hadrons.

\footnotetext{
*e-mail: lebedev@iastate.edu
} 


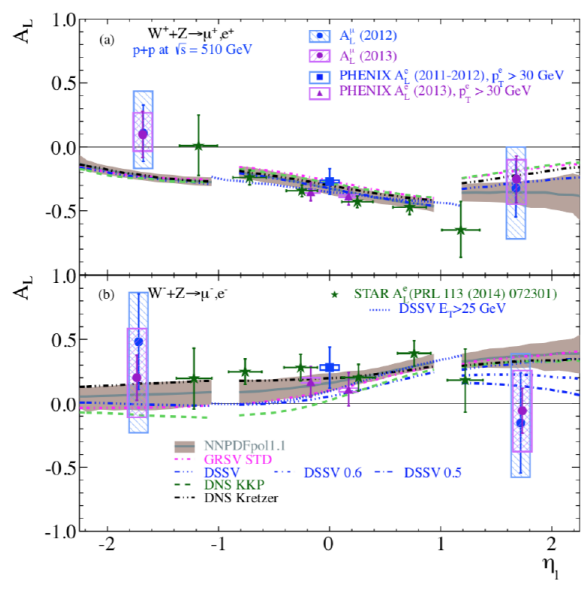

Figure 1. Longitudinal single spin asymmetry of $W$-bosons as a function of pseudorapidity.

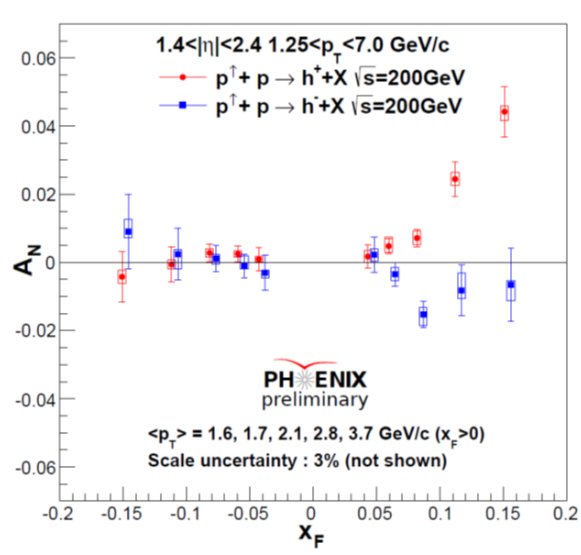

Figure 2. Transverse single spin asymmetry of charged hadrons in $p+p$ collisions as a function of $x_{F}=2 p_{T} / \sqrt{s}$.

It is interesting to compare these results with $A_{N}$ observed in $p+A$ collisions, since this may reveal the influence of cold nuclear matter effects like nuclear shadowing, gluon saturation and multiple scattering on $A_{N}$. Transverse single spin asymmetry for charged hadrons produced in $p+A$ collisions is shown in Fig. 4.These results were published in [2] and reveal significantly reduced asymmetries in proton-nucleus collisions. There is a clear and strong dependence both on nuclear size $\left(A^{1 / 3}\right)$ and centrality of the collisions $\left(N_{\text {coll }}\right)$.
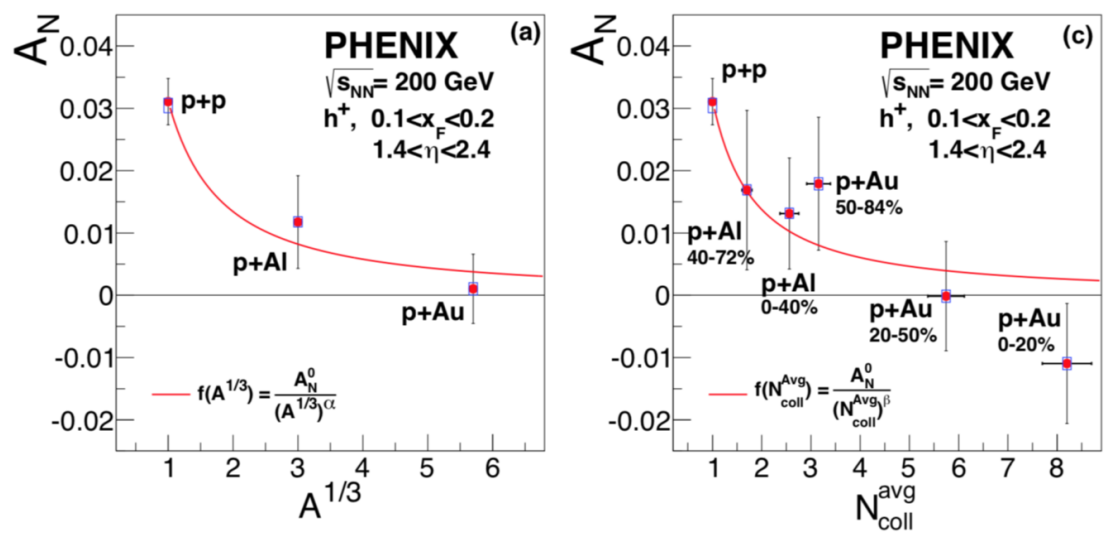

Figure 3. Transverse single spin asymmetry of charged hadrons produced in $\mathrm{p}+\mathrm{A}$ collisions as a function of target size (left) and centrality (right).

Transverse single spin asymmetry of $J / \psi$ 's produced in $p+A$ collisions is shown in Fig. 3 . The results were measured at forward (proton-going) and backward (nucleus-going) rapidity, and published in [5]. All data points are consistent with zero, except for $p+A u$ collisions at low $p_{T}$ both for forward and backward rapidity.

Transverse single spin asymmetry for $\eta$-mesons produced in $p+p$ collisions is shown in Fig. 5 and Fig. 6 as a function of transverse momentum. The results are consistent with 

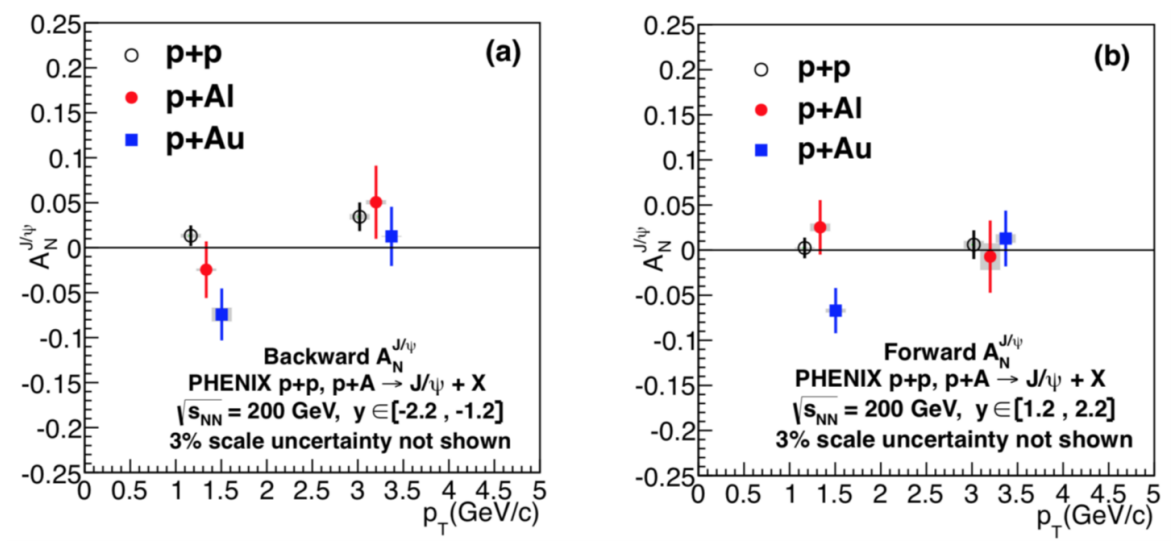

Figure 4. Transverse single spin asymmetry of $J / \psi$ produced in $p+p$ and $p+A$ collisions as a function of transverse momentum for backward (left) and forward (right) rapidities.

zero, but there is a noticeable structure as a function of $p_{T}$. The new results (5) are a huge improvement over previously published PHENIX results (Fig. 6).

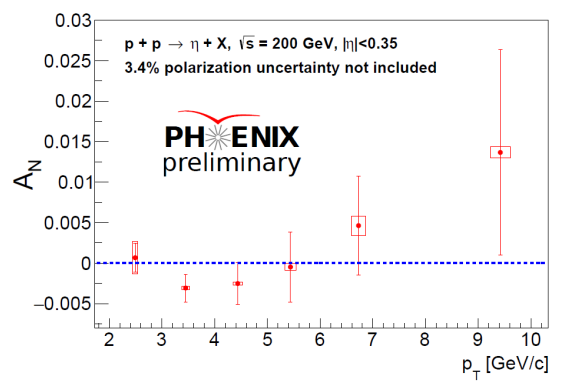

Figure 5. Transverse single spin asymmetry of $\eta$-mesons as a function of $p_{T}$.

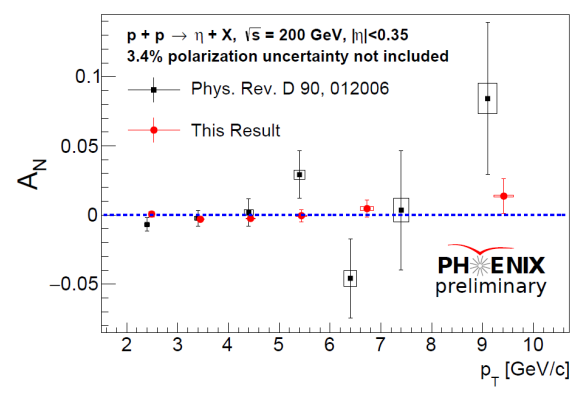

Figure 6. Transverse spin asymmetry of $\eta$ mesons as a function of $p_{T}$.

\section{Small systems}

Strongly asymmetric nuclear collisions like $p+A u$ or ${ }^{3} H e+A u$ provide a good tool for understanding particle production mechanisms, as well as studying the interactions among these particles after their initial creation in the collision.

Charged particle density as a function of pseudorapidity is shown in Fig. 7 for four different colliding species. Both wounded-quark model [3] and 3D hydrodynamics model [4] describe the data reasonably well.

Flow of charged particles produced in the same four systems is shown in Fig. 8. These results were published in [6]. 3D hydrodynamics model describes data well except for ${ }^{3} \mathrm{He}+$ $A u$ collisions at forward rapidity where it overpredicts the data.

Hydrodynamic models can be tested by controlling the geometry of the collisions. $p+A u$, $d+A u$ and ${ }^{3} \mathrm{He}+\mathrm{Au}$ collisions have different elliptical and triangular excentricities $\left(\varepsilon_{2}\right.$ and 


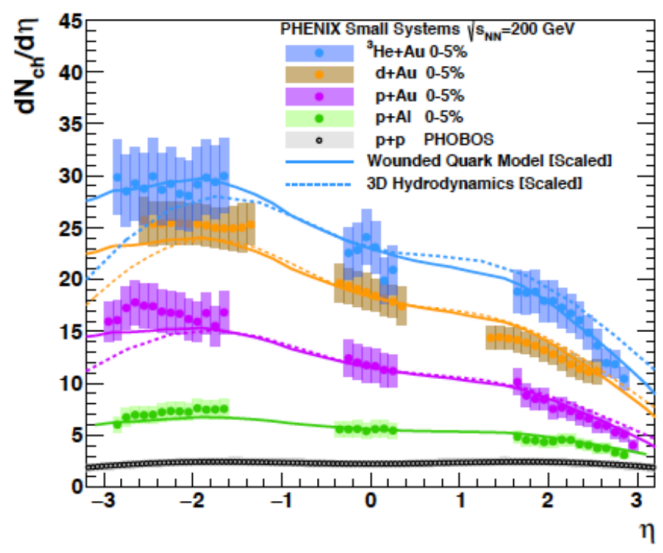

Figure 7. Charged particle density as a function of pseudorapidity for $p+A l, p+A u, d+A u$ and ${ }^{3} H e+A u$ collisions along with wounded-quark model [3] and 3D hydrodynamics model [4] predictions.

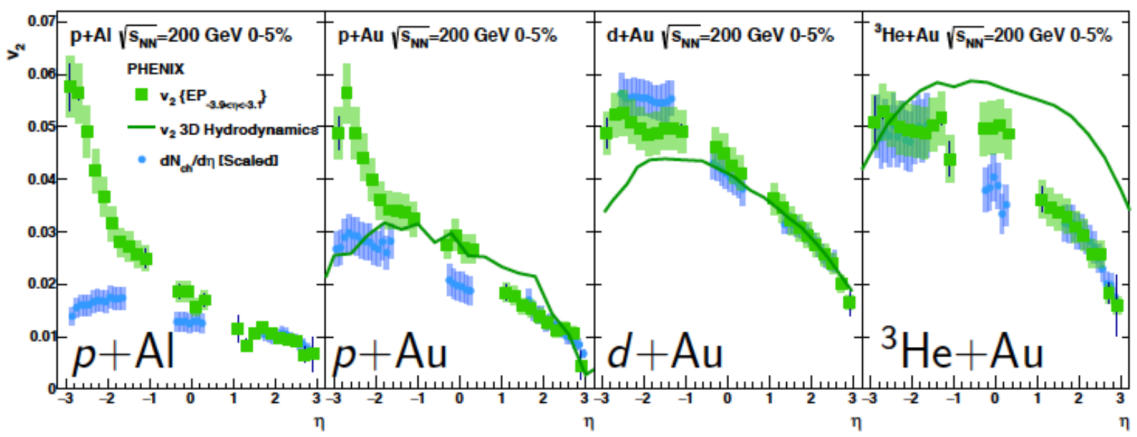

Figure 8. Charged particle flow as a function of pseudorapidity for $p+A l, p+A u, d+A u$ and ${ }^{3} H e+A u$ collisions. Green curve is a prediction from 3D hydrodynamics model [4].

$\left.\varepsilon_{3}\right)$, which should translate into momentun flow $\left(v_{2}\right.$ and $\left.v_{3}\right)$. Comparing flow in these theree colliding systems can provide a valuable tool for testing hydrodynamic models.

Eccentricities calculated for $p+A u, d+A u$ and ${ }^{3} \mathrm{He}+A u$ collisions are shown on Fig. 9. As one can see, elliptic eccentricity $\varepsilon_{2}$ is relatively small in $p+A u$ while relatively large and almost equal for $d+A u$ and ${ }^{3} \mathrm{He}+A u$. Triangular eccentricity $\varepsilon_{3}$ is relatively large for ${ }^{3} \mathrm{He}+\mathrm{Au}$ sytem while relatively smaller and almost equal for the other two colliding systems. This is confirmed in Fig. 10, where time evolution of these three colliding syetems is shown according to SONIC model [7].

Elliptic and triangular flow for the three colliding systems is shown in Fig. 11. As one can see, the ordering of both $v_{2}$ and $v_{3}$ follows the ordering of eccentricities $\varepsilon_{2}$ and $\varepsilon_{3}$. This means that regardles of the flow mechanism, the correlation is geometrical in nature.

Measurement of the nuclear modification factor in proton-nucleus or duteron-nucleus collisions provides an important tool for separating cold nuclear matter effects from QGP formation signatures. The PHENIX experiment has recently measured nuclear modification factor of $J / \psi$ in $p+A l, p+A u$ and ${ }^{3} \mathrm{He}+\mathrm{Au}$ collisions at forward and backward rapidities us- 


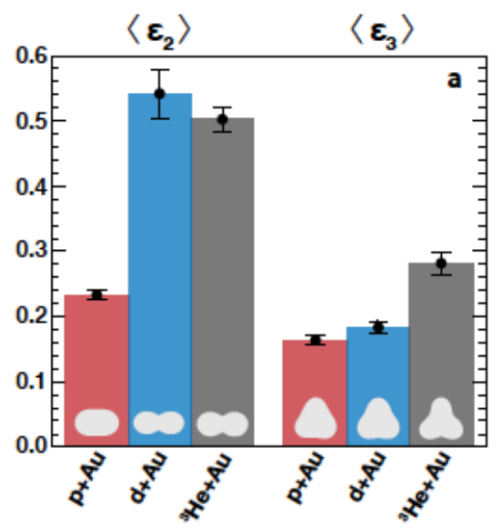

Figure 9. Eccentricities calculated for $p+A u$, $d+A u$ and ${ }^{3} \mathrm{He}+\mathrm{Au}$ collisions.

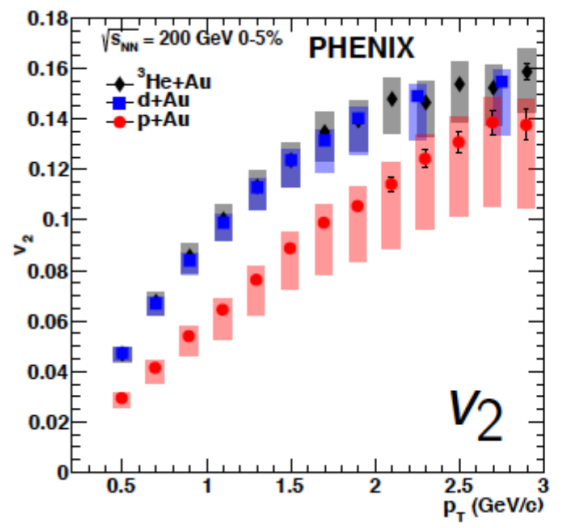

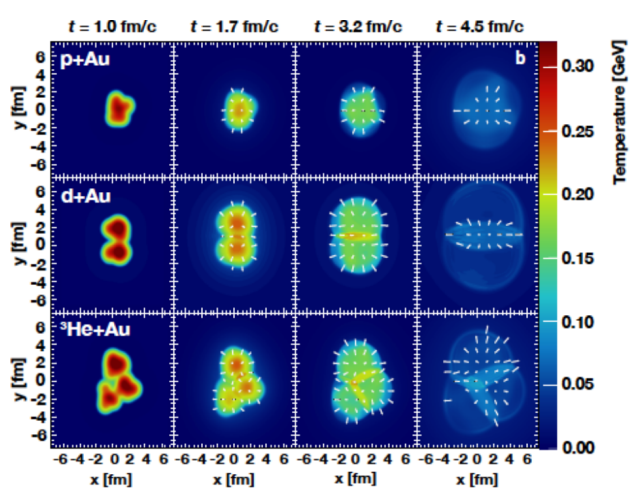

Figure 10. Time evolution of $p+A u, d+A u$ and ${ }^{3} \mathrm{He}+\mathrm{Au}$ collisions according to SONIC model [7].

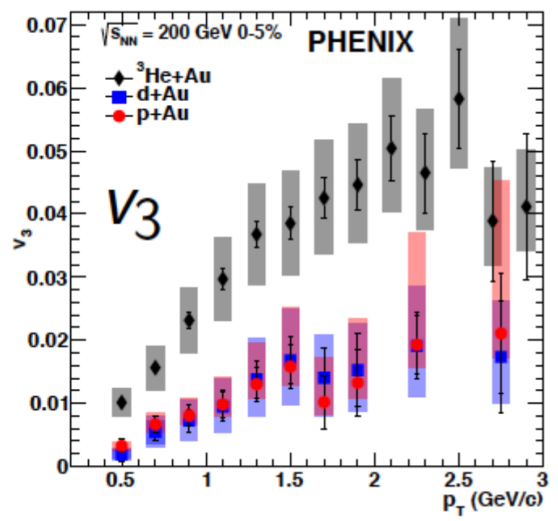

Figure 11. Charged particle flow as a function of $p_{T}$ for $p+A u, d+A u$ and ${ }^{3} H e+A u$ collisions. Left plot: elliptic flow $v_{2}$, right plot: triangular flow $v_{3}$.

ing PHENIX Muon Arms. The results are presented in Fig. 12 as a function of transverse momentum.

$p+A l$ collisions show, within experimental uncertainty, no modification relative to $p+p$ collisions. In $p+A u$ collisions, however, there is a clear indication of suppression at low $p_{T}$ both at forward and backward rapidity. Comparison of $p+A u$ and ${ }^{3} \mathrm{He}+\mathrm{Au}$ results show no dependence on the projectile size.

Using asimuthal correlations of $\mu \mu$ pairs from semileptonic heavy flavor decays, the PHENIX experiment conducted a mesurement of Drell-Yan pair spectra as a function of mass and transverse momentum. This measurement is based on a novel analysis technique using both unlike-sign and like-sign $\mu \mu$ pairs to separate charm, bottom and Drell-Yan contributions. Drell-Yan spectra in $\mathrm{p}+\mathrm{p}$ collisions are well described by PYTHIA and NLO claculations.

In addition, the PHENIX experiment made similar measurement in $p+A u$ collisions. whicjh allowed us to calculate nuclear modification factor for Drell-Yan pairs in $p+A u$ 

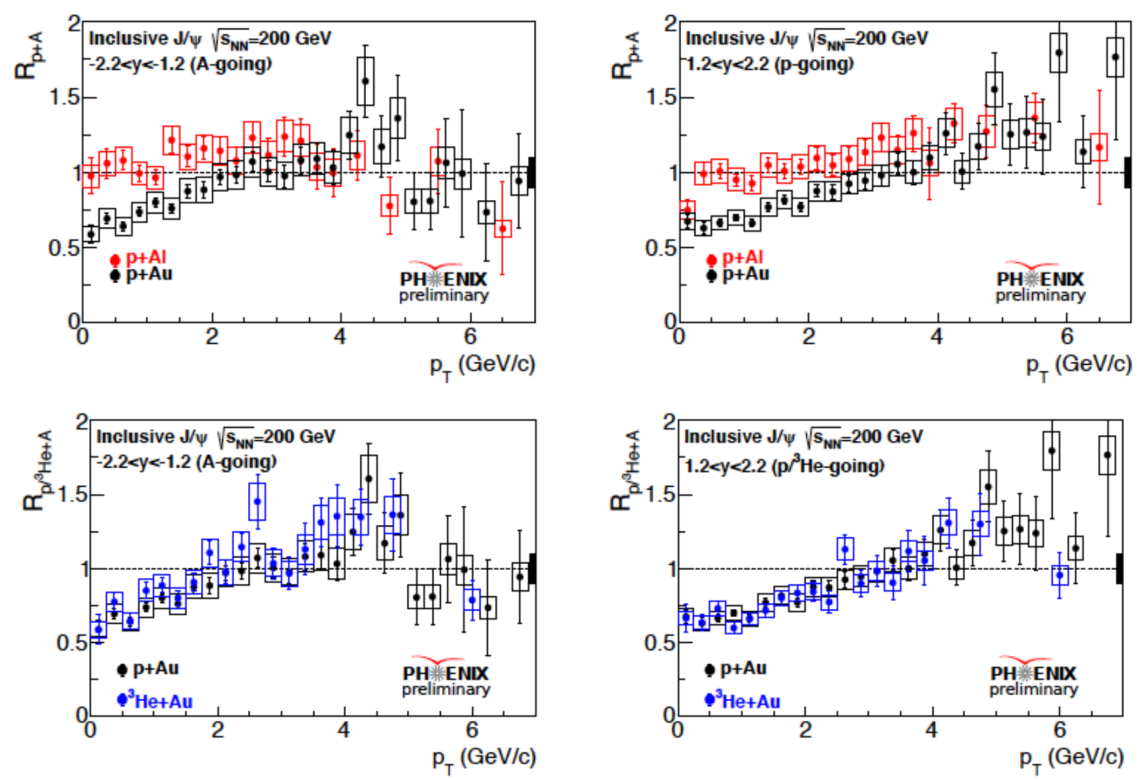

Figure 12. $R_{p A u}$ of $J / \psi$ as a function of $p_{T}$.

collisions. The results are presented in Fig. 13 as a function of pair mass and transverse momentum. Within experimental uncertainties the results are consistent with no modification, but at forward rapidity (proton-going direction) there is an indication of enhancement at high $p_{T}$.

The PHENIX experiment measured direct photon yields in $A u+A u$ collisions at different energies. The results are shown in Fig. 14 as a function of charged particle density $\left(d N_{c h} / d \eta\right)$, along with the results from other experiments and a fit to $p+p$ data scaled by number of binary collisions $\left(N_{\text {coll }}\right)$. For all energies and colliding species direct photon yiled in nucleus-nucleus collisions shows significant enhancement relative to $p+p$. Direct photon yield is found to be proportional to $\left(d N_{c h} / d \eta\right)^{\alpha}$ where $\alpha=1.25$. This scaling behavior holds for a wide range of beam energies from $39 \mathrm{GeV} /$ nucl. to $2760 \mathrm{GeV} /$ nucl. This suggests that the main sources of direct photons are very similar for all beam energies.

It is interestig to note that direct photon yield in $p+A u$ and $d+A u$ collisions, also shown in Fig. 14 smoothly fills the gap between $\mathrm{p}+\mathrm{p}$ and nucleus-nucleus collisions.

Fig. 15 shows nuclear modification factor $R_{d A u}$ for direct photons produced in $p+A u$ collisions as a function of transverse momentum. Although the uncertainty of the measurement is large, there seems to be a significant enhancement of direct photon yield in $d+A u$ collisions relative to $p+p$ collisions. A comparison with a theoretical prediction of thermal direct photon production [8], suggests that direct thermal photons are produced even in $p+A u$ collisions.

\section{Heavy lons}

A summary of PHENIX measurements of the nuclear modification factors for different particle species in $\mathrm{Au}+\mathrm{Au}$ collisons is shown in Fig. 16. Several features can be noted in this plot. Photons are almost unmodified, baryons are not suppressed at intermediate $p_{T}$, while mesons 

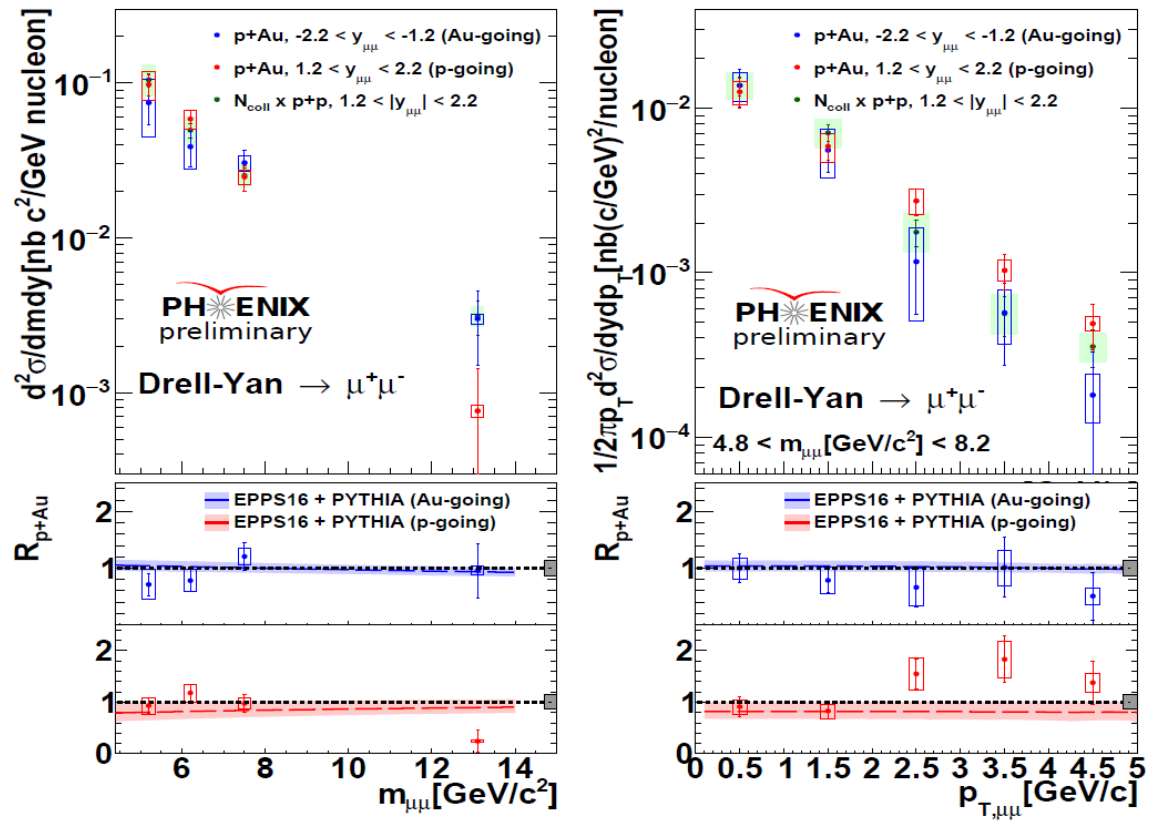

Figure 13. Drell-Yan pair production cross-section in $p+A u$ collisions as a function of pair mass (left) and $p_{T}$ (right). The inserts at the bottom show $p+A$ to $p+p$ ratios.

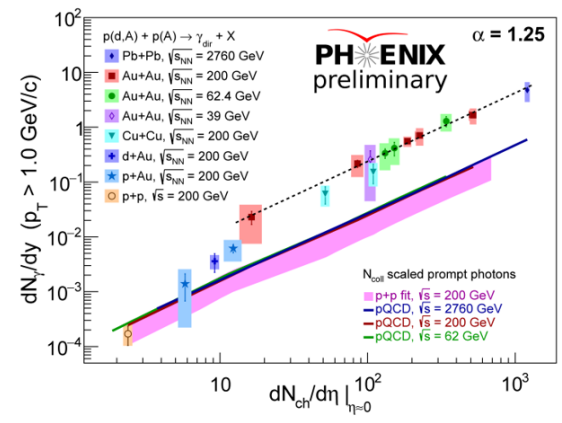

Figure 14. Direct photon yield as a function of charged particle density.

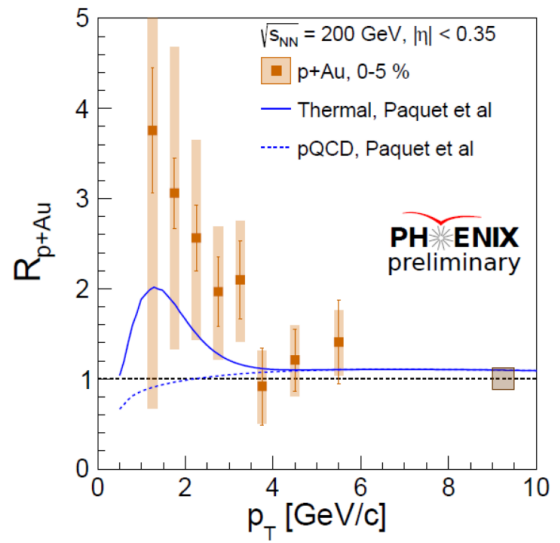

Figure 15. Nuclear modification factor for direct photons produced in $p+A u$ collisions as a function of $p_{T}$.

are strongly suppressed. An outlier in this pictrure is $\phi$-meson, which, at intermediate $p_{T}$ is suppressed between baryons and mesons. The reason for this could be either strangeness content or difference in mass.

To clarify this question it is useeful to look at suppression for other strange mesons and mesons with similar mass. Fig. 17 shows nuclear modification factor for different particle species in $\mathrm{Cu}+\mathrm{Au}$ collisions as a function of transverse momentum. $\phi$-meson is an outlier 
again at low transverse momentum, but $K_{S}$ and $\omega$ mesons follow the trend of $\pi^{0}$ and $\eta$ mesons at high $p_{T}$.

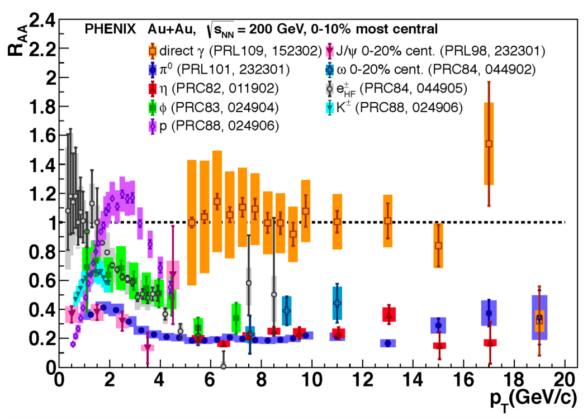

Figure 16. A summary of PHENIX measurements of the nuclear modification factors for different particle species in $A u+A u$ collisons.

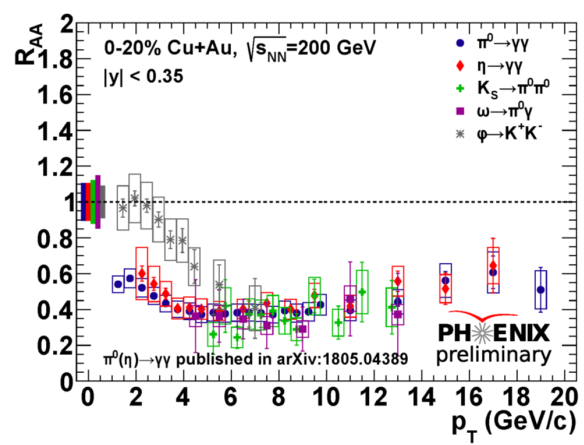

Figure 17. Nuclear modification factor for different particle species in $C u+A u$ collisions.

Another comparison of $\phi$ and $\omega$ mesons is shown in Fig. 18 and Fig. 19. Fig. 18 shows $\phi$-meson nuclear modification factor in $\mathrm{Cu}+\mathrm{Cu}, \mathrm{Cu}+\mathrm{Au}$, and $\mathrm{Au}+\mathrm{Au}$ collisions as a function of transverse momentum for centralities corresponding to approximately the same number of participants $N_{\text {part }}$, while Fig. 19 shows the same dependence for $\omega$-meson. Both $\omega$ and $\phi$ mesons behave similarly in all colliding systems when the same number of participants is selected.

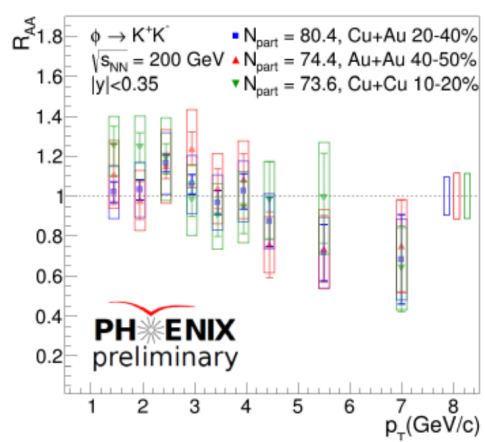

Figure 18. $\phi$-meson nuclear modification factor in $C u+C u, C u+A u$, and $A u+A u$ collisions.

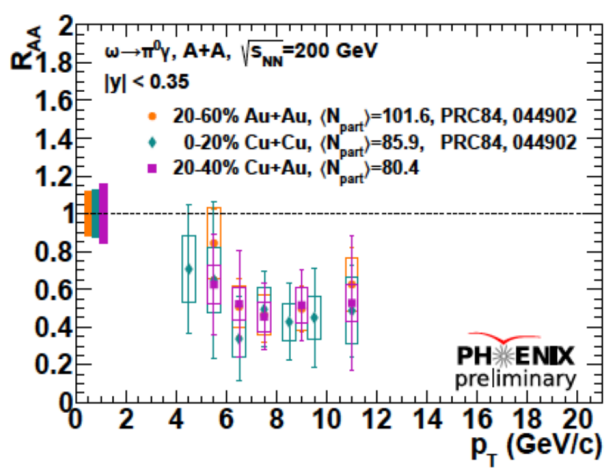

Figure 19. $\omega$-meson nuclear modification factor in $C u+C u, C u+A u$, and $A u+A u$ collisions.

The PHENIX experiment recently published a new and improved measurement of heavy flavor electrons in $\mathrm{p}+\mathrm{p}$ collisions [9]. The measurement based on unfolding analysis where the yield of open heavy flavor hadrons is determined from the measured inclusive heavy flavor electron spectrum, and the electron distance of closest approach (DCA) distribution measured using PHENIX silicon vertex detector.

The yields of charm and bottom electrons are found to be consistent with FONLL predictions. This measurement will provide a new precision baseline for an improved reesults on heavy flavor suppression in heavy ion collisions.

In addition, an improvement of analysis techniques allowed us to make the first measurement of heavy flavor flow in Au+Au collisons. Fig. 20 and Fig. 21 show elliptic flow of 
electrons from charm and bottom decays correspondingly. The plots also show the elliptic flow of charged hadrons also measured by PHENIX. Charm appears to flow less than light flavor hadrons. The uncertainty on the bottom flow measurement is large, but there is an indication that bottom flows too.

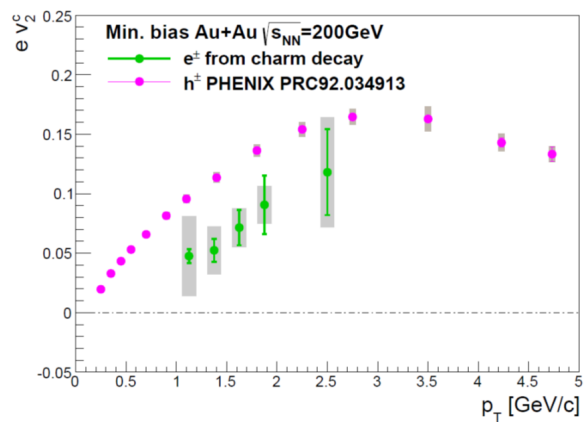

Figure 20. Charm elliptic flow $v_{2}$ as a function of $p_{T}$.

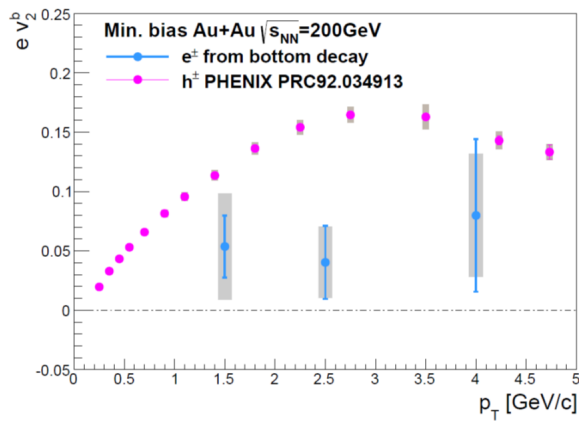

Figure 21. Bottom elliptic flow $v_{2}$ as a function of $p_{T}$.

\section{Conclusions}

The PHENIX experiment has published longitudinal single spin asymmetry measurement of $W$-bosons. The results are in agreement with STAR results and world fits.

Transverse single spin asymmetry for charged hadrons in $p+p$ collisions shows statistically significant deviation from zero at positive $x_{F}$, which is not expected in conventional pQCD calculations.

Transverse single spin asymmetry for charged hadrons in $p+A$ collisions shows clear dependence on nuclear target size $\left(A^{1 / 3}\right)$ and centrality $\left(N_{\text {coll }}\right)$.

New results on $\eta$-meson transverse single spin asymmetry are a great improvement on previous PHENIX measurement. $A_{N}$ is consistent with zero, but exibits a noticeable structure.

Recently published $J / \psi$ transverse single spin asymmetry measurement illustrates importance of changing nuclear target in spin physics. In $p+A u$ collisions at low transverse momentum a deviation from zero is observed.

Comprehensive set of measurements of longitudinal dynamics published by the PHENIX experiment provides a good support for wounded-quark and 3D hydrodynamics model.

Small systems geometry scan results published in Nature Physics. Hydrodynamic models describe the data well. Ordering of elliptic and triangular flow in $p+A u, d+A u$ and ${ }^{3} \mathrm{He}+\mathrm{Au}$ collisions follows the ordering of the corresponding eccentricities.

$J / \psi$ nuclear modification in $p / d /{ }^{3} \mathrm{He}+A$ collisions indicates that modification depends on target size, but not projectile size.

Modification of $\phi$-meson in small systems is very similar to that of $\pi^{0}$ despite differences in mass and strangeness content.

The PHENIX experiment performed the first measurement of Drell-Yan in small systems at RHIC. There is a hint of enhancement at high transverse momentum in proton-going direction, but no firm conclusions can be made due to large experimental uncertainty.

Photon enhancement in small systems is an important additional evidence in support of QGP droplet formation in small systems. 
In $A u+A u$ collisions photons are unmodified, baryons slightly enhanced at intermediate $p_{T}$, while mesons are strongly suppressed. $\phi$-meson is an outlier with suppression between that of baryons and mesons.

Single particle nuclear modification factor is independent of collision species when selecting for similar number of participants.

For nuclear modification factor strangeness is important at low transverse momentum, but not a high.

The PHENIX experiment performed a measurement of charm and bottom flow in $A u+A u$ collisions. This is the first measurement of bottom flow at RHIC energies. Charm flows less than light quarks. There is an indication of bottom flow, but experimental uncertainties are too high to make a definitive conclusion. 


\section{References}

[1] C.Aidala et al.,, Phys. Rev. D 86, 032007 (2018)

[2] C.Aidala et al., arXiv:1903.07422 (submitted to PRL).

[3] M. Barej, A. Bzdak, and P. Gutowski, Phys. Rev. C 97, 034901, (2018)

[4] P. Bozek and W. Broniowski, Phys. Lett. B 739, 308 (2014)

[5] C.Aidala et al., Phys. Rev. D 98, 012006 (2018)

[6] A.Adare et al., Phys. Rev. Lett. 121, 222301 (2018)

[7] M. Habich, J. L. Nagle, and P. Romatschke, Eur. Phys. J. C 7515 (2015).

[8] Chun Shen, Jean-François Paquet, Gabriel S. Denicol, Sangyong Jeon, and Charles Gale, Phys.Rev. C 95, 014906 (2017).

[9] C.Aidala et al., Phys. Rev. D 99, 092003 (2019) 